FERMILAB-TM-2029

\title{
Minimizing the Pacman Effect
}

\author{
D. Ritson \\ Stanford University \\ Stanford, California 94305 \\ W. Chou \\ Fermi National Accelerator Laboratory \\ P.O. Box 500, Batavia, Illinois 60510
}

October 1997 


\section{Disclaimer}

This report was prepared as an account of work sponsored by an agency of the United States Government. Neither the United States Government nor any agency thereof, nor any of their employees, makes any warranty, expressed or implied, or assumes any legal liability or responsibility for the accuracy, completeness, or usefulness of any information, apparatus, product, or process disclosed, or represents that its use would not infringe privately owned rights. Reference herein to any specific commercial product, process, or service by trade name, trademark, manufacturer, or otherwise, does not necessarily constitute or imply its endorsement, recommendation, or favoring by the United States Government or any agency thereof. The views and opinions of authors expressed herein do not necessarily state or reflect those of the United States Government or any agency thereof.

\section{Distribution}

Approved for public release; further dissemination unlimited. 
FERMILAB-TM-2029

October 1997

\title{
MINIMIZING THE PACMAN EFFECT
}

\author{
D. Ritson, Physics Dept, Stanford Univ, Stanford, CA 94305, USA \\ W. Chou, Fermilab, P.O. Box 500, Batavia, IL 60510, USA
}

\begin{abstract}
The Pacman bunches will experience two deleterious effects: tune shift and orbit displacement. It is known that the tune shift can be compensated by arranging crossing planes $90^{\circ}$ relative to each other at successive interaction points (IPs). This paper gives an analytical estimate of the Pacman orbit displacement for a single as well as for two crossings. For the latter, it can be minimized by using equal phase advances from one IP to another. In the $\mathrm{LHC}$, this displacement is in any event small and can be neglected.
\end{abstract}

\section{Introduction}

It is believed that the attainable beam-beam tune shifts in hadron colliders may not be limited by bunches in a standard environment, but by bunches that circulate past "gaps" in the counter circulating beam, the so called Pacman bunches. Such bunches will suffer tune shifts and orbit displacements different from the "average" bunches. Therefore if the machine is optimized for average bunches the Pacman bunches will not be in an optimized environment and will suffer enhanced losses. However loss of a Pacman bunch will create new Pacman bunches in the counter circulating beam, and over the course of time holes will develop in both beams and eventually the beams may be destroyed. A circulating bunch encounters the identical counter circulating bunches at points separated by half the circumference. It is then possible to compensate the tune shift differences by arranging for cancellation between the two points. For example, in the LHC, this is easily accomplished for equal $\beta_{i p}^{*}$ ( $\beta$-function at the IP) by crossings planes at $90^{\circ}$ relative to each other at the two high luminosity IPs 1 and 5. Below we examine whether by an appropriate choice of phase advance between the IPs we can also achieve cancellation for the orbit displacements. Obviously if the crossings are successively in horizontal and vertical planes no cancellation are possible. However if two horizontal or vertical crossing planes are used, or if the probably superior tilted plane geometry is used, there is an optimum phase difference that minimizes but doesn't cancel the orbit displacements at the IPs. This phase advance is calculated in the following section. Not, perhaps, surprisingly it corresponds to equal phase advances between the two IPs for the two half sectors of the machine. We also calculate the displacement for a single horizontal or vertical crossing and find that it gives an almost identical displacement to that found for two crossings symmetrically related in phase. A further point should be mentioned. Bunches that do not encounter an opposing head-on bunch collision should be 
quite stable. Therefore the gaps in the counter circulating beam which straddle the IP are not destructive and only gaps that lie on one side or the other of the IP are destructive. (However a bunch that does not suffer a head-on collision with the counter circulating beam does not contribute to the luminosity and therefore even if stable is of little interest.)

Finally we examine the physical magnitude of the expected orbit effect in the LHC. We find them to be relatively small compared with the degradation of the beam-beam limit due to the additional Pacman tune spread.

\section{Theoretical Derivation}

The derivation uses a number of straightforward properties for finding eigen solutions for the equilibrium orbit in a machine. We assume a machine with two identical IRs, $A$ and $B$, at the start and midpoint of the circumference around the machine. The IPs are at $\alpha$ (slope of the $\beta$-function) equal to zero, and are assumed to have equal $\beta_{i p}^{*}$. In units of $x / \beta_{i p}^{*}$ the transfer matrix $R$ around the machine from IP A back to IP A is given by

$$
R=\left(\begin{array}{cc}
\cos (\mu) & \sin (\mu) \\
-\sin (\mu) & \cos (\mu)
\end{array}\right)
$$

where $\mu$ is the phase shift around the machine. We shall use the shorthand notation

$$
R=M(\mu)
$$

and this has the standard property that

$$
M(\mu) M(\phi)=M(\mu+\phi)
$$

Orbit displacements are only significant for bunches that suffer head-on collisions. The missing "Pacman gap" in the counter circulating beam can then be encountered before collision ("IN" configuration) or after collision ("OUT" configuration). In a single passage across the IR to the IP the gap will cause the bunch to deviate from its equilibrium orbit by a deflection as it passes the missing bunch(es). To a good approximation the missing parasitic crossings are $90^{\circ}$ in phase away from the IP and the net effect is for there to be a transverse displacement at the IP and a close to zero angular displacement. In units of the net displacement (see subsection 2.4 below) we can represent the single pass displacement as a vector, $V$,

$$
\begin{aligned}
V & \simeq\left(\begin{array}{l}
1 \\
\epsilon
\end{array}\right) \\
\epsilon & \simeq \beta_{i p}^{*} / L
\end{aligned}
$$

where $L$ is the distance of the missing bunch from the IP.

We assume that the IPs are separated in phase by $\phi$. We will give a detailed derivation first for the IN case and for the equilibrium displacement $x$ at IP A (the first IP), which is defined as

$$
X=\left(\begin{array}{l}
x \\
\theta
\end{array}\right)
$$


Then in matrix notation the eigen solution for $X$ is found by equating the $X$ at each sequential turn with the $X$ at the previous turn. We then find

$$
X=M(\mu) X+M(\mu-\phi) V+V
$$

This can be rearranged as

$$
(M(0)-M(\mu)) X=M(\mu-\phi) V+M(0) V
$$

$X$ is then specified by

$$
X=(M(0)-M(\mu))^{-1}(M(\mu-\phi)+M(0)) V
$$

\subsection{Evaluation of Orbit Displacement $X$}

The previous result can be simplified by noting that the term

$$
M(0)-M(\mu)=\left(\begin{array}{cc}
1-\cos (\mu) & -\sin (\mu) \\
\sin (\mu) & 1-\cos (\mu)
\end{array}\right)
$$

or

$$
M(0)-M(\mu)=2 \sin \left(\frac{\mu}{2}\right)\left(\begin{array}{cc}
\sin \left(\frac{\mu}{2}\right) & -\cos \left(\frac{\mu}{2}\right) \\
\cos \left(\frac{\mu}{2}\right) & \sin \left(\frac{\mu}{2}\right)
\end{array}\right)
$$

Taking the inverse

$$
(M(0)-M(\mu))^{-1}=\frac{1}{2 \sin \left(\frac{\mu}{2}\right)}\left(\begin{array}{cc}
\sin \left(\frac{\mu}{2}\right) & \cos \left(\frac{\mu}{2}\right) \\
-\cos \left(\frac{\mu}{2}\right) & \sin \left(\frac{\mu}{2}\right)
\end{array}\right)
$$

This in turn can be written as

$$
(M(0)-M(\mu))^{-1}=\frac{-1}{2 \sin \left(\frac{\mu}{2}\right)} M\left(-\frac{\mu}{2}-\frac{\pi}{2}\right)
$$

Substituting this into Eq. (9) we get

$$
X=\frac{-1}{2 \sin \left(\frac{\mu}{2}\right)}\left(M\left(\frac{\mu}{2}-\phi-\frac{\pi}{2}\right)+M\left(-\frac{\mu}{2}-\frac{\pi}{2}\right)\right) V=S V
$$

where $S$ is a matrix defined by the above equation.

A small change in the angular component, $\theta$, of $X$ has an insignificant effect on the deflection at the IP, and the only quantity of interest is the spatial component, $x$, of $X$. Remembering $V$ is approximated by Eq. (4) and therefore

$$
x \simeq S_{11}+\epsilon S_{12}
$$

or substituting we obtain the simple and final result

$$
x \simeq \frac{-1}{2 \sin \left(\frac{\mu}{2}\right)}\left(-\sin \left(\frac{\mu}{2}\right)+\sin \left(\frac{\mu}{2}-\phi\right)-\epsilon\left(\cos \left(\frac{\mu}{2}\right)+\cos \left(\frac{\mu}{2}-\phi\right)\right)\right)
$$




\subsection{Results for Two Crossings}

The previous subsection evaluated the offset at IP A for a gap in parasitic crossings on the incoming side of the IP. There are four cases, incoming and outgoing gaps and IPs A and $B$. We summarize the basic equations and solutions for the four cases. The results are evaluated for same sign crossing angles at the two IPs. The four additional cases for opposite sign crossings can be found by replacing $\phi$ by $\phi+\pi$. These additional cases are degenerate to an interchange of IPs A and B and therefore do not modify the following results.

IN for IP A

$$
X=(M(0)-M(\mu))^{-1}(M(\mu-\phi)+M(0)) V
$$

IN for IP B

$$
X=(M(0)-M(\mu))^{-1}(M(\phi)+M(0)) V
$$

OUT for IP A

$$
X=(M(0)-M(\mu))^{-1}(M(\mu)+M(\mu-\phi)) V
$$

OUT for IP B

$$
X=(M(0)-M(\mu))^{-1}(M(\mu)+M(\phi)) V
$$

The results evaluated for these cases are

$$
\begin{aligned}
x_{\text {in } A} & \simeq \frac{-1}{2 \sin \left(\frac{\mu}{2}\right)}\left(-\sin \left(\frac{\mu}{2}\right)+\sin \left(\frac{\mu}{2}-\phi\right)-\epsilon\left(\cos \left(\frac{\mu}{2}\right)+\cos \left(\frac{\mu}{2}-\phi\right)\right)\right) \\
x_{\text {in } B} & \simeq \frac{-1}{2 \sin \left(\frac{\mu}{2}\right)}\left(-\sin \left(\frac{\mu}{2}\right)-\sin \left(\frac{\mu}{2}-\phi\right)-\epsilon\left(\cos \left(\frac{\mu}{2}\right)+\cos \left(\frac{\mu}{2}-\phi\right)\right)\right) \\
x_{\text {out } A} & \simeq \frac{-1}{2 \sin \left(\frac{\mu}{2}\right)}\left(+\sin \left(\frac{\mu}{2}\right)+\sin \left(\frac{\mu}{2}-\phi\right)+\epsilon\left(\cos \left(\frac{\mu}{2}\right)+\cos \left(\frac{\mu}{2}-\phi\right)\right)\right) \\
x_{\text {out } B} & \simeq \frac{-1}{2 \sin \left(\frac{\mu}{2}\right)}\left(+\sin \left(\frac{\mu}{2}\right)-\sin \left(\frac{\mu}{2}-\phi\right)+\epsilon\left(\cos \left(\frac{\mu}{2}\right)+\cos \left(\frac{\mu}{2}-\phi\right)\right)\right)
\end{aligned}
$$

Using the approximation that $\epsilon$ is small, to order $\epsilon$ terms in $\cos$ can be neglected. We then obtain the following simple results.

$$
x_{i n A}+x_{i n B} \simeq+1
$$

and

$$
\boldsymbol{x}_{\text {out } A}+\boldsymbol{x}_{\text {out } B} \simeq-1
$$

Equivalent, for either the in or out case to

$$
\left|x_{A}\right| \text { and } / \text { or }\left|x_{B}\right| \geq \frac{1}{2}
$$


For the symmetric case where $\phi=\frac{\mu}{2}$

$$
\left|x_{A}\right|=\left|x_{B}\right|=\frac{1}{2}
$$

The symmetric case represents the optimum configuration.

\subsection{Results for a Single IP}

If the crossing planes are $90^{\circ}$ relative to each other, horizontal or vertical, then the orbit displacements in the two planes are orthogonal and we need only consider displacements from one plane. For instance assuming horizontal crossing for IP A and vertical crossing for IP B the horizontal orbit displacement at IP A will be given at A from

$$
X_{A}=M(\mu) X_{A}+M(0) V
$$

equivalent to

$$
X_{A}=(M(0)-M(\mu))^{-1} V
$$

or evaluating as in the previous subsection

$$
x_{A}=\frac{1}{2}\left(1+\epsilon \cdot \cot \left(\frac{\mu}{2}\right)\right)
$$

or neglecting terms in $\epsilon$

$$
x_{A} \simeq \frac{1}{2}
$$

The corresponding displacement at $\mathrm{B}$ is

$$
X_{B}=M\left(\frac{\mu}{2}\right) X_{A}
$$

and again evaluating as before for equal phases $\mathrm{A}$ to $\mathrm{B}$ and $\mathrm{B}$ to $\mathrm{A}$

$$
X_{B}=\frac{-1}{2 \sin \left(\frac{\mu}{2}\right)} M\left(\frac{\mu}{2}\right) M\left(-\frac{\mu}{2}-\frac{\pi}{2}\right) V
$$

or equivalently

$$
x_{B}=\frac{\epsilon}{2 \sin \left(\frac{\mu}{2}\right)} \simeq 0
$$

Thus again in units of V the deflection at the IPs is one half, identical to the previous result.

\subsection{Numerical Results}

The units of $\mathrm{V}$ are easily evaluated for the LHC. The orbit displacement at the IP for a single pass is $[1]$

$$
\Delta x_{s}=\frac{8 \pi N_{p} \Delta \nu_{h o}}{\theta_{\text {cross }}}
$$

where $\Delta x_{s}$ is in units of $\sigma_{x}$ (the rms beam transverse size), $\Delta \nu_{h o}$ is the head-on tune shift per IP, $\theta_{\text {cross }}$ is the full crossing angle in units of $\sigma_{x^{\prime}}$ (the $r m s$ beam angular size), and $N_{p}$ 
is the number of parasitic crossings. For multipasses the displacement with equal phasing between IPs is $\frac{1}{2} \Delta x_{s}$.

We approximate $N_{p}$ as being equal to the number of crossing points from the IP to an effective point in the separation magnets, namely eighteen effective crossings. Orbit displacement only destroys bunches that collide head-on with the counter circulating beam, and thus a worst case is for all the bunches in the counter circulating beams to be missing either prior or after the IP. If destructive bunch loss required all bunches to be missing on one side of the IP, then only a single bunch would be lost in the counter circulating beam (insufficient to destabilize the circulating beam) and the beams would remain stable after the loss of a single bunch. To propagate, destructive bunch loss must occur when at least half the bunches are missing, i.e., for a propagating Pacman effect the bunches must be unstable for $N_{p} \leq 9$.

Using a $\beta_{i p}$ of $50 \mathrm{~cm}$, an emittance of $5 \times 10^{-8} \mathrm{~cm}-\mathrm{rad}$, a $\theta_{\text {cross }}$ of $200 \mu \mathrm{rad}$, a $\Delta \nu_{h o}$ of 0.0034 per IP, and $N_{p}$ equal to 9 , the spatial component of $\frac{1}{2} V$ (the symmetric case) is $0.06 \sigma_{x}$ or $1 \mu \mathrm{m}$ for a beam with a $\sigma_{x}$ of $16 \mu \mathrm{m}$. Such an orbit displacement is very small and will contribute minimally to instability. For all practical purposes it is negligible and the orbit displacement will not contribute to any appreciable extent to a Pacman effect. Of course the additional tune spread from Pacman bunches, comparable to the head-on beam beam tune shift does play a major role and successive crossing planes rotated by $90^{\circ}$ will be very helpful.

Herr has previously investigated the impact of the Pacman effect on LHC running. [2] Our results agree with his with the exception of our distinction of a localized loss of bunches (where we agree with Herr) and a runaway Pacman effect, where we require a factor two times higher threshold. Herr points out that if more IPs than IPs 1 and 5 are run simultaneously at high luminosity the Pacman orbit effects are substantial and might require a bunch by bunch feedback control system. However it is presently envisaged that high LHC luminosity running will only occur simultaneously for IPs 1 and 5 . Therefore with symmetric phasing both our and Herr's results show feedback control of Pacman orbit displacements will be unnecessary.

\section{Conclusions}

The cases of interest described above are for successive horizontal or vertical crossings at the high luminosity IPs, or for $45^{\circ}$ tilted crossings. For Pacman bunches the most important deleterious effects are associated with tune shifts. The Pacman tune shift effects are eliminated by using crossing planes $90^{\circ}$ relative to each other, and therefore in all probability the candidates for crossing geometries are "horizontal/vertical" or tilted planes. The horizontal/vertical case is not modified by interferences between the two IPs and therefore for a given tune all phase advances between IPs are equally acceptable. For the tilted planes the optimum choice to minimize orbit excursions is to use equal phase advances from IPs $1 \rightarrow 5$ and from IPs $5 \rightarrow 1$. 
In any event the orbit displacement contribution to Pacman instability is small and, even for a $200 \mu \mathrm{rad}$ "worst case" crossing angle, can for all effective purposes be regarded as negligible and close to zero.

\section{References}

[1] John Irwin, SSC-233 (1989).

[2] Werner Herr, LHC Project Report 39, CERN (1996). 